\title{
Strangeness production in high-multiplicity events
}

\author{
Marat Siddikov@ and Iván Schmidt@ \\ Departamento de Física, Universidad Técnica Federico Santa María, \\ y Centro Científico-Tecnológico de Valparaíso, Casilla 110-V, Valparaíso, Chile
}

(Received 7 January 2021; accepted 1 July 2021; published 26 July 2021)

\begin{abstract}
In this paper we analyze in detail the production of strangeness in proton-proton collisions in the kinematics of large transverse momenta $p_{T}$ of produced hadrons. Using the color dipole framework, we estimate the production cross sections for kaons and demonstrate that the shapes of the $p_{T}$ dependence are in agreement with available experimental data. We also analyze the self-normalized yields of strange hadrons as a function of multiplicity of coproduced hadrons, and find that the predictions are in agreement with the faster-than-linear growth seen in experimental data. Our description is largely parameter free and extends our previous studies dedicated to the explanation of multiplicity enhancement of quarkonia, as well as open heavy flavor $D$ and $B$ mesons.
\end{abstract}

DOI: $10.1103 /$ PhysRevD.104.016024

\section{INTRODUCTION}

Since the early experiments at RHIC and SPS [1-6], the production of strangeness in hadronic collisions has been used as one of the probes of quark-gluon plasma (QGP) formation and, therefore, described in the framework of QGP-inspired hydrodynamic models [7-21]. Usually the QGP manifests itself indirectly in experimental data, and for this reason, in order to disentangle it, properly designed observables are required. Historically, enhancement of strange particle yields in heavy-ion collisions (compared to $p A$ and $p p$ production of the same strange hadrons) has been used for this purpose. However, this observable requires careful understanding of strangeness production in two channels, as well as possible contributions of cold nuclear matter effects in heavy-ion collisions. Another observable, which is usually attributed to QGP, is the enhancement of strangeness in events with large multiplicity of coproduced hadrons. This observable might be studied independently in $p p, p A$, or $A A$ collisions. While early experiments confirmed the expected enhancement in heavy-ion collisions, similar effects were later observed at the LHC in not only heavy-ion but also $p A[22,23]$ and even $p p$ collisions [24], where QGP formation in significant amounts is highly unlikely even at TeV-range collision energies. For this reason it makes sense to better understand the microscopic mechanisms of this phenomenon, at least in $p p$ collisions. In general, the application of perturbation

Published by the American Physical Society under the terms of the Creative Commons Attribution 4.0 International license. Further distribution of this work must maintain attribution to the author(s) and the published article's title, journal citation, and DOI. Funded by SCOAP ${ }^{3}$. theory for strangeness production is challenging due to the lack of a hard scale. Therefore, the phenomenological description of strangeness production has been mostly limited to studies in the framework of Monte Carlo generators $[25,26]$, and inevitably includes additional modeldependent assumptions.

Recently, detailed studies of heavy quarkonia [27-29] and open heavy flavor mesons [30] in $p p$ collisions have discovered that a similar enhancement with multiplicity also happens for the production of heavier flavors. This effect has a quite complicated dependence on the details of experimental setup, on the existence of rapidity gaps between the heavy hadrons and colliding protons [31], as well as (possibly) on the quantum numbers of the produced quarkonia states [32]. Taking into account the similarity of multiplicity enhancements observed in the strange, charm, and bottom sectors, it is very desirable to describe the phenomenon for all flavors in the same framework. Since the strange quarks have very light masses, in general it is not possible to apply the theoretical tools that rely on the heavy quark mass limit for their justification. Nevertheless, in the kinematics of very large transverse momenta $p_{T}$ of produced strange hadrons, the latter variable effectively plays the role of a hard scale, partially justifying the use of such perturbative tools. For this reason, in what follows we will try to extend the earlier description of the charm sector to the strangeness production in large- $p_{T}$ kinematics. Our analysis will be mostly focused on the production of kaons and $\Lambda$ baryons, due to the lack of information about the fragmentation functions of other strange hadrons.

While the high-energy hadroproduction can be described in the two-gluon (two-Pomeron) fusion picture [33-43], as was pointed out in Ref. [25], the description of the 
multiplicity dependence presents challenges for the established two-Pomeron paradigm. These findings agree with expectations of the saturation phenomena in the small- $x$ kinematics, as well as earlier results of the Regge approach [44-49], which relate the multiplicity enhancement to contributions of multiple Pomeron exchanges. In the framework of the color dipole (CGC/saturation) approach [50-58], all such multigluon (multi-Pomeron) interactions are naturally incorporated. This approach gives a plausible description of different heavy mesons, particularly the $D$ - and $B$-meson production [59-63]. The generalization of this framework to high-multiplicity events is well known from the literature [63-72] and allows to explain the multiplicity dependence in both the charm and bottom sectors [29,62,73-78]. For this reason, in what follows we will use the CGC/saturation approach for our evaluations.

The paper is structured as follows. In Sec. II we introduce the framework for strangeness production in the CGC/saturation approach. In Sec. III we make numerical estimates for the cross sections and compare them with available experimental data. In Sec. IV we discuss the multiplicity dependence of strange hadrons in the large- $p_{T}$ kinematics and demonstrate that our approach can describe the experimentally observed dependence for kaons and $\Lambda$ baryons. Finally, in Sec. V we draw conclusions.

\section{PRODUCTION OF STRANGE HADRONS VIA FRAGMENTATION}

We assume that all strange hadrons are produced via a fragmentation mechanism, and we will perform our evaluations within the framework developed earlier in Refs. [59-63]. In this approach the cross section is related to the quark pair $\bar{Q} Q$ production cross section by

$\frac{d \sigma_{p p \rightarrow M+X}}{d y d^{2} p_{T}}=\sum_{i} \int_{x_{Q}}^{1} \frac{d z}{z^{2}} D_{i}\left(\frac{x_{Q}(y)}{z}\right) \frac{d \sigma_{p p \rightarrow \bar{Q}_{i} Q_{i}+X}}{d y^{*} d^{2} p_{T}^{*}}$,

where $y$ is the rapidity of the produced strange hadron, $y^{*}=y-\ln z$ is the rapidity of the quark, $p_{T}$ is the transverse momentum of the produced strange hadron, $D_{i}(z)$ is the fragmentation function which describes the formation of a given final state from a parton of flavor $i$, and $d \sigma_{p p \rightarrow \bar{Q}_{i} Q_{i}+X} / d y^{*}$ is the cross section of quark pair production with quark rapidity $y^{*}$ and transverse momentum $p_{T}^{*}=p_{T} / z$. In what follows we will focus on the production of kaons and $\Lambda$ baryons, whose fragmentation functions are well known from the literature (see Appendix A for details). Naturally, the dominant contribution in the strange sector stems from the strange quarks, although there are also contributions from other flavors. In what follows we will focus on the evaluation of the cross section $d \sigma_{p p \rightarrow \bar{Q}_{i} Q_{i}+X} / d y^{*} d^{2} p_{T}^{*}$, which appears in the integrand of Eq. (1).

In the rest frame of one of the protons, this process might be viewed as a fluctuation of the incoming virtual gluon

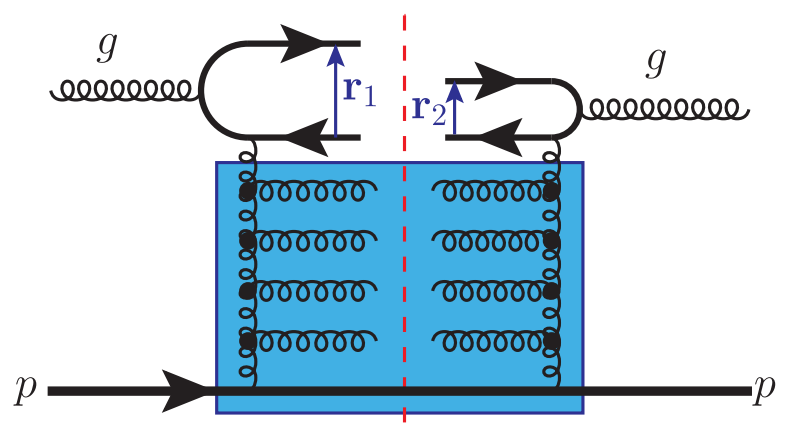

FIG. 1. Color dipole picture of the quark hadroproduction in gluon-proton collisions. The colored square block in the interior part of the diagram contains all possible multigluon (multiPomeron) interactions which are included in the dipole amplitude. The vertical dashed line stands for unitarity cuts. It is assumed that the produced quark $Q$ in the amplitude and its conjugate are projected onto the eigenstate with definite momentum $\boldsymbol{p}_{T}$ and later fragment into the open heavy flavor meson.

into a heavy $\bar{Q} Q$ pair, with subsequent scattering of the $\bar{Q} Q$ dipole on the target proton, as shown in Fig. 1. In the LHC kinematics the gluon densities are enhanced, and thus it is not appropriate to speak about dipole interactions mediated by the exchange of individual gluons or Pomerons in the $t$ channel. For this reason we will use for our evaluations the color dipole framework (also known as CGC/saturation) [50-58], which naturally incorporates all possible multigluon (multi-Pomeron) interactions. At high energies, the color dipoles are eigenstates of the interaction, and therefore they can be used as universal elementary building blocks, automatically accumulating both the hard and soft fluctuations [79]. In fact, the light-cone color dipole framework has been successfully applied to phenomenological descriptions of both hadron-hadron and leptonhadron collisions [80-87]. Another advantage of the CGC/ saturation framework is that it allows a relatively straightforward extension for the description of high-multiplicity events, as discussed in Refs. [63,66-72].

In the dipole approach, the quark production cross section might be represented as a convolution of a gluon wave function with a linear combination of dipole amplitudes $[61,63]$ (see also Appendix B),

$$
\begin{aligned}
& \frac{d \sigma_{p p \rightarrow \bar{Q}_{i} Q_{i}+X}(y, \sqrt{s})}{d y d^{2} p_{T}} \\
& =\int d^{2} k_{T} x_{1} g\left(x_{1}, \boldsymbol{p}_{T}-\boldsymbol{k}_{T}\right) \int_{0}^{1} d z \int_{0}^{1} d z^{\prime} \\
& \times \int \frac{d^{2} r_{1}}{4 \pi} \int \frac{d^{2} r_{2}}{4 \pi} e^{i\left(r_{1}-r_{2}\right) \cdot \boldsymbol{k}_{T} \Psi_{\bar{Q} Q}^{\dagger}}\left(r_{2}, z, p_{T}\right) \Psi_{\bar{Q} Q}\left(r_{1}, z, p_{T}\right) \\
& \times N_{M}\left(x_{2}(y) ; \vec{r}_{1}, \vec{r}_{2}\right), \\
& x_{1,2} \approx \frac{\sqrt{m_{M}^{2}+\left\langle p_{\perp M}^{2}\right\rangle}}{\sqrt{s}} e^{ \pm y},
\end{aligned}
$$


where $y$ and $\boldsymbol{p}_{T}$ are the rapidity and transverse momenta of the produced strange quark in the center-of-mass frame of the colliding protons, $\boldsymbol{k}_{T}$ is the transverse momentum of the strange quark with respect to the incident gluon, $g\left(x_{1}, \boldsymbol{p}_{T}\right)$ in the first line of Eq. (2) is the unintegrated parton distribution function (uPDF) of gluon, and $\Psi_{\bar{Q} Q}(r, z)$ is the $\bar{Q} Q$ component of the gluon light-cone wave function, with transverse separation between quarks $r$ and the light- cone fraction of the quark $z$. In general, this is a nonperturbative object, and there is no model-independent way to evaluate it [88]. For this reason, in what follows we will restrict our consideration to the kinematics of large transverse momenta of produced hadrons, which in view of Eq. (2) implies that typical sizes of the dipoles are also small, $\langle r\rangle \sim 1 / p_{T}$. In this kinematics we may use standard perturbative expressions [89,90],

$$
\begin{gathered}
\Psi_{T}^{\dagger}\left(r_{2}, z, Q^{2}\right) \Psi_{T}\left(r_{1}, z, Q^{2}\right)= \\
\frac{\alpha_{s} N_{c}}{2 \pi^{2}}\left\{\epsilon_{f}^{2} K_{1}\left(\epsilon_{f} r_{1}\right) K_{1}\left(\epsilon_{f} r_{2}\right)\left[e^{i \theta_{12}} z^{2}+e^{-i \theta_{12}}(1-z)^{2}\right]\right. \\
\left.+m_{f}^{2} K_{0}\left(\epsilon_{f} r_{1}\right) K_{0}\left(\epsilon_{f} r_{2}\right)\right\}, \\
\Psi_{L}^{\dagger}\left(r_{2}, z, Q^{2}\right) \Psi_{L}\left(r_{1}, z, Q^{2}\right)=\frac{\alpha_{s} N_{c}}{2 \pi^{2}}\left\{4 Q^{2} z^{2}(1-z)^{2} K_{0}\left(\epsilon_{f} r_{1}\right) K_{0}\left(\epsilon_{f} r_{2}\right)\right\}, \\
\epsilon_{f}^{2}=z(1-z) Q^{2}+m_{f}^{2}, \\
\left|\Psi^{(f)}\left(r, z, Q^{2}\right)\right|^{2}=\left|\Psi_{T}^{(f)}\left(r, z, Q^{2}\right)\right|^{2}+\left|\Psi_{L}^{(f)}\left(r, z, Q^{2}\right)\right|^{2} .
\end{gathered}
$$

The meson production amplitude $N_{M}$, as was shown in Refs. $[57,58,61,62]$ (see also a brief derivation in Appendix B), might be represented as

$$
\begin{aligned}
N_{M}\left(x, \vec{r}_{1}, \vec{r}_{2}\right)= & -\frac{1}{2} N\left(x, \vec{r}_{1}-\vec{r}_{2}\right)-\frac{1}{16}\left[N\left(x, \vec{r}_{1}\right)+N\left(x, \vec{r}_{2}\right)\right]-\frac{9}{8} N\left(x, \bar{z}\left(\vec{r}_{1}-\vec{r}_{2}\right)\right) \\
& +\frac{9}{16}\left[N\left(x, \bar{z} \vec{r}_{1}-\vec{r}_{2}\right)+N\left(x, \bar{z} \vec{r}_{2}-\vec{r}_{1}\right)+N\left(x, \bar{z} \vec{r}_{1}\right)+N\left(x, \bar{z} \vec{r}_{2}\right)\right],
\end{aligned}
$$

where $N(x, \overrightarrow{\boldsymbol{r}})$ is the color-singlet dipole scattering amplitude. This result was obtained under the implicit assumption that dipoles are small, which is justified in the large- $p_{T}$ kinematics. A more accurate expression, which takes into account possible quadrupole contributions, was found in Ref. [91]. However, in view of the complexity of the latter result, as well as the expected smallness of the quadrupole contributions for small dipoles, in what follows we will use for our estimates the simpler expression (8). In the LHC kinematics at large transverse momenta (our principal interest), the natural choice of the saturation scale is $\mu_{F} \sim p_{T}$, which significantly exceeds the saturation scale $Q_{s}(x)$. This finding justifies the use of twoPomeron approximation. However, in the kinematics of smaller $p_{T}$, potentially there could be multi-Pomeron contributions, like those shown in the right panel of Fig. 1.
We will not consider such contributions since, as we mentioned earlier, we cannot describe the small- $p_{T}$ region due to the lack of the nonperturbative photon wave function $\Psi_{\bar{Q} Q}$.

The uPDF of gluon, which appears in the prefactor of Eq. (2), can be related to the integrated PDF $x G\left(x, \mu_{F}\right)$ as [92]

$$
x g\left(x, k^{2}\right)=\left.\frac{\partial}{\partial \mu_{F}^{2}} x G\left(x, \mu_{F}\right)\right|_{\mu_{F}^{2}=k^{2}},
$$

and the natural choice of the factorization scale $\mu_{F} \sim p_{T}$ significantly exceeds the saturation scale $Q_{s}(x)$. In this kinematics it is possible to relate the gluon densities to the dipole scattering amplitude $N(y, r)=\int d^{2} b N(y, r, b)$ via a set of identities [66,93],

$$
\begin{gathered}
\frac{C_{F}}{2 \pi^{2} \bar{\alpha}_{S}} N(y, \vec{r})=\int \frac{d^{2} k_{T}}{k_{T}^{4}} \phi\left(y, k_{T}\right)\left(1-e^{i \vec{k}_{T} \cdot \vec{r}}\right), \quad x G\left(x, \mu_{F}\right)=\int_{0}^{\mu_{F}} \frac{d^{2} k_{T}}{k_{T}^{2}} \phi\left(x, k_{T}\right), \\
x G\left(x, \mu_{F}\right)=\frac{C_{F} \mu_{F}}{2 \pi^{2} \bar{\alpha}_{S}} \int d^{2} r \frac{J_{1}\left(r \mu_{F}\right)}{r} \nabla_{r}^{2} N(y, \vec{r}) .
\end{gathered}
$$




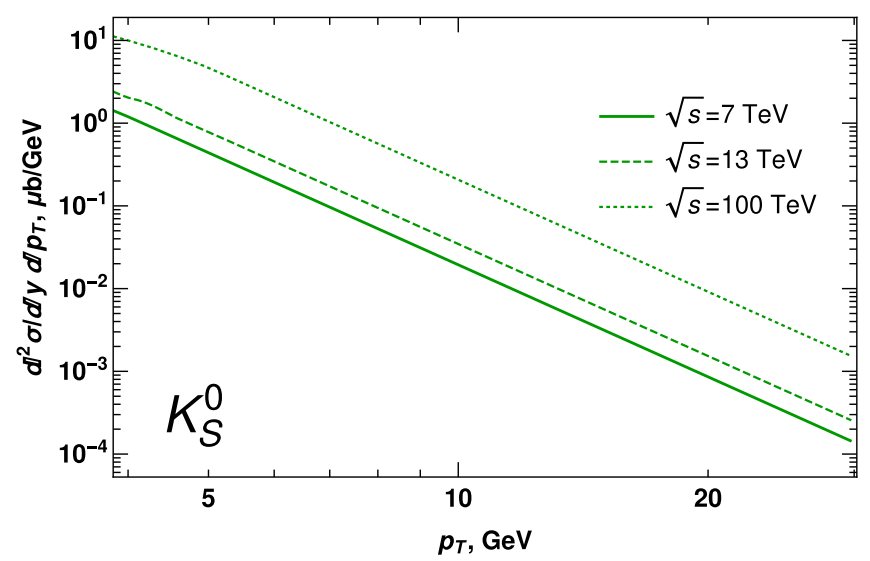

FIG. 2. $p_{T}$ dependence of the cross section $d \sigma / d p_{T}$ for $K_{S^{-}}^{0}$ meson production at central rapidities, evaluated with the twoPomeron fusion mechanism. Results for $\Lambda$ have a similar shape, due to the similarity of their fragmentation functions (see details in Appendix A).
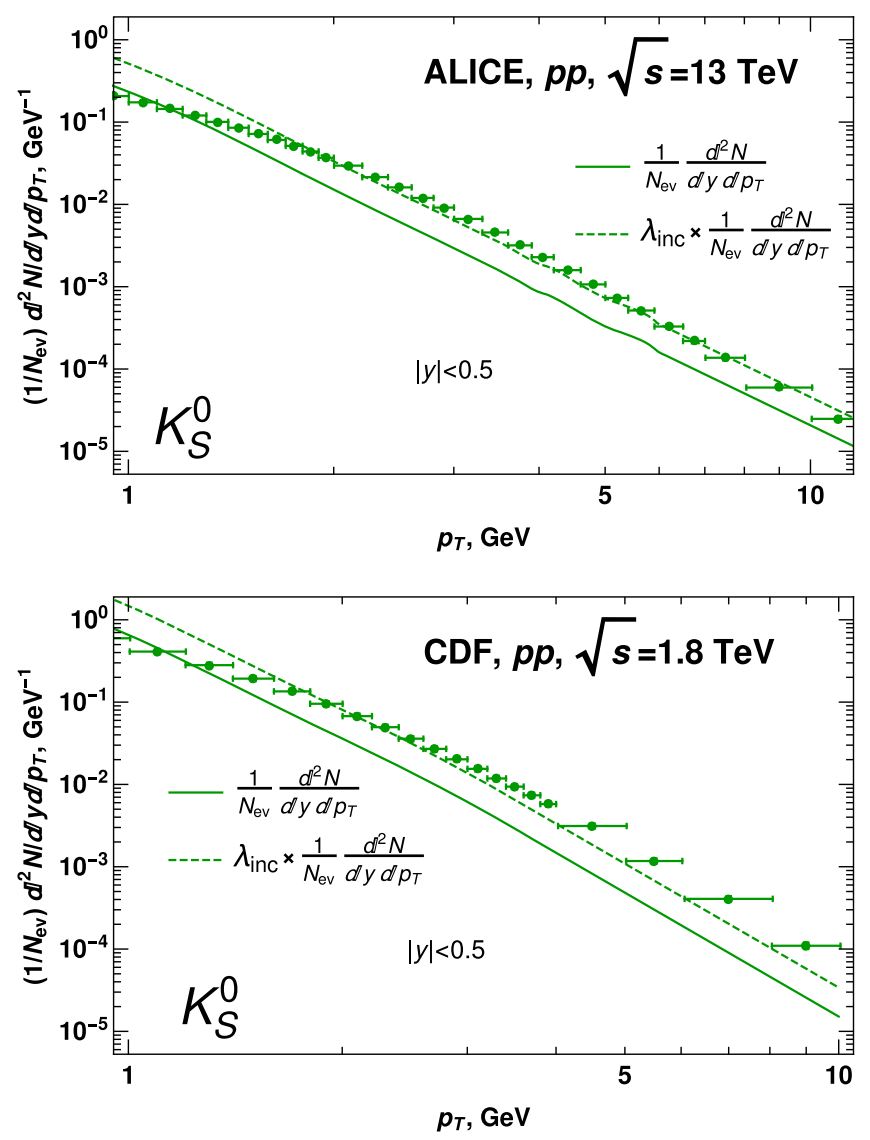

This allows to rewrite the result in a symmetric and selfconsistent form, which allows straightforward generalization for high-multiplicity events.

\section{NUMERICAL RESULTS}

For the sake of definiteness, in our numerical evaluations we will take the " $b$-CGC" parametrization of the dipole cross section proposed and analyzed in detail in Refs. [94-98],

$N(x, \overrightarrow{\boldsymbol{r}})=\sigma_{0} \times \begin{cases}N_{0}\left(\frac{r Q_{s}(x)}{2}\right)^{2 \gamma_{\mathrm{eff}}(r)}, & r \leq \frac{2}{Q_{s}(x)}, \\ 1-\exp \left(-\mathcal{A} \ln \left(\mathcal{B} r Q_{s}\right)\right), & r>\frac{2}{Q_{s}(x)},\end{cases}$

$\mathcal{A}=-\frac{N_{0}^{2} \gamma_{s}^{2}}{\left(1-N_{0}\right)^{2} \ln \left(1-N_{0}\right)}, \quad \mathcal{B}=\frac{1}{2}\left(1-N_{0}\right)^{-\frac{1-N_{0}}{N_{0} \gamma_{s}}}$,
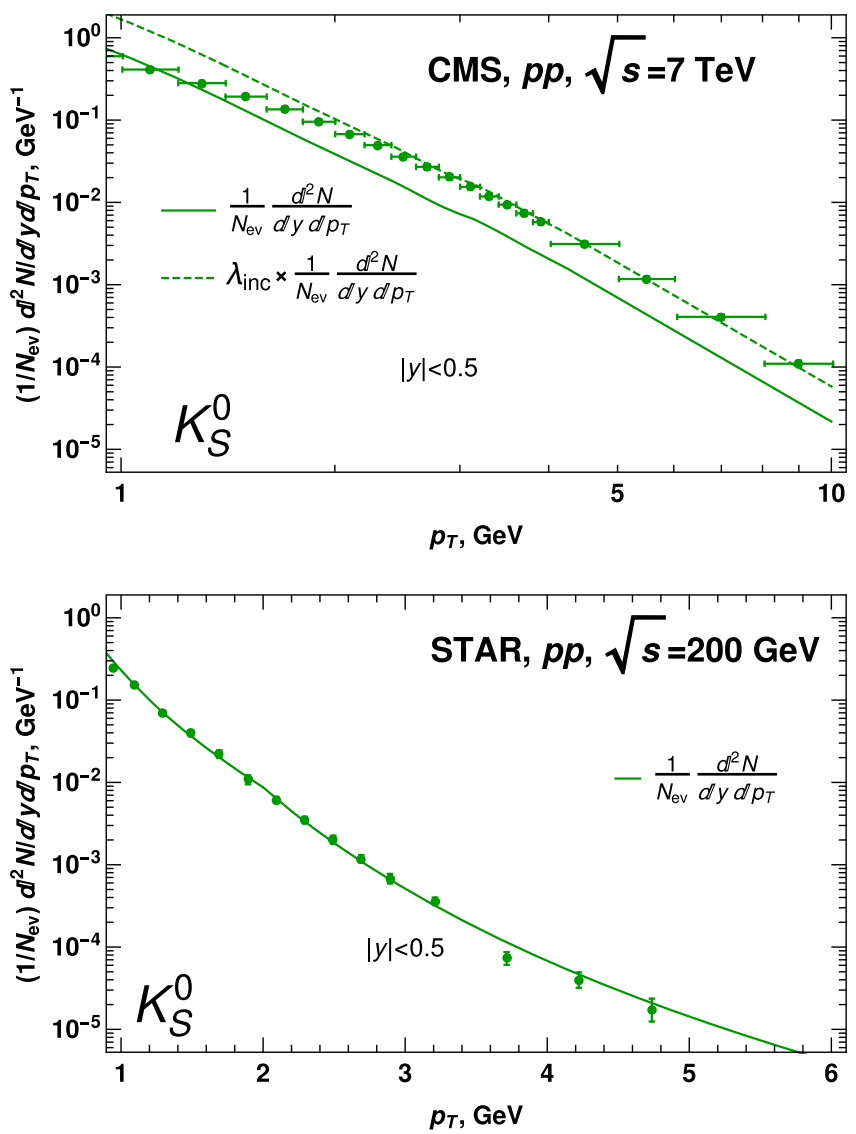

FIG. 3. Self-normalized $p_{T}$ dependence of the $K_{S}^{0}$-meson yields at central rapidities. Theoretical predictions (solid line) are compared with experimental data from the ALICE [100], CMS [101], CDF [102], and STAR [103] collaborations. As we explain in the text, our approach is not very reliable at small $p_{T}$, and thus the evaluation of the global normalization factor $N_{\mathrm{ev}}$ might have large nonperturbative corrections. In order to demonstrate that the description of the shape is correct, we also plot the yields multiplied by a constant factor $\lambda_{\text {inc }} \approx 1.7-2.2$ (dashed lines). 


$$
\begin{gathered}
Q_{s}(x)=\left(\frac{x_{0}}{x}\right)^{\lambda / 2}, \quad \gamma_{\mathrm{eff}}(r)=\gamma_{s}+\frac{1}{\kappa \lambda Y} \ln \left(\frac{2}{r Q_{s}(x)}\right), \\
\gamma_{s}=0.762, \quad \lambda=0.2319 \\
\sigma_{0}=21.85 \mathrm{mb}, \quad x_{0}=6.2 \times 10^{-5},
\end{gathered}
$$

which is widely used in the literature. For the fragmentation functions of kaons and $\Lambda$ baryons we will use the parametrizations discussed in Appendix A. The fragmentation functions for $K^{ \pm}$and $K_{S}^{0}$ are constrained by the isospin symmetry relation [99]

$$
D_{i}^{K_{S}^{0}}\left(z, \mu^{2}\right)=\frac{1}{2} D_{\bar{i}}^{K^{ \pm}}\left(z, \mu^{2}\right),
$$

and for this reason the cross sections for $K_{S}^{0}$ and $K^{ \pm}$ production are proportional to each other with very good precision. The fragmentation function of $\Lambda$ baryons available from Ref. [99] in general is different from that of kaons. However, as shown in Appendix A, in the large- $z$ domain, which gives the dominant contribution, the strange flavor component of the fragmentation function is approximately proportional to that of kaons. Therefore, we may expect that the $\Lambda$-baryon cross section is proportional to that of kaons in the large- $p_{T}$ kinematics. For this reason, in what follows we will focus on the discussion of the $K_{S}^{0}$ production cross section.

In the left panel of Fig. 2 we show the results for $K_{S}^{0}$ production cross section in the kinematics of ongoing and planned experiments. In the literature, all of the experimental papers contain results for the self-normalized yields $N_{\mathrm{ev}}^{-1} d N / d y d p_{T}$, instead of cross sections. The normalization parameter $N_{\mathrm{ev}}$ is chosen as the total number of events $N_{\mathrm{ev}} \sim \int d p_{T} d N / d p_{T}$ or the number of non-singlediffractive events $N_{\text {NSD }}$. This normalization coefficient gets its dominant contribution from the small- $p_{T}$ region and thus cannot be evaluated reliably for light quarks in our approach. This introduces a normalization uncertainty in our evaluations of such self-normalized yields. In Fig. 3 we compare the calculated yields with available experimental data from the ALICE [100], CMS [101], CDF [102], and STAR [103] collaborations [104]. The STAR data [103] were included by the authors of Ref. [99] in their global fit of fragmentation functions, and for this reason the description of these data is nearly perfect. For data from LHC and Tevatron, the model provides a very reasonable description of the shape, although, as expected, there is a mismatch in the normalization by a factor of 2 . The theoretical shape of the $p_{T}$ dependence starts deviating from the experimental data in the region $p_{T} \lesssim 2 \mathrm{GeV}$, where nonperturbative effects become pronounced.
In view of these findings, we believe that our approach might be used for the evaluation of observables that get a dominant contribution from the region of large $p_{T} \gtrsim 2 \mathrm{GeV}$.

\section{MULTIPLICITY DEPENDENCE}

Since the dipole approach provides a reasonable description of the strangeness production in the large- $p_{T}$ kinematics, we can apply it to the study of the dependence on the number of charged particles $N_{\mathrm{ch}}$ coproduced together with a given strange hadron.

The studies of high-multiplicity events were started more than 40 years ago in Refs. [44-49], using the Regge approach. Starting from unitarity and very general properties of particle-Reggeon vertices, the enhancement of multiplicity in final states was predicted as one of the manifestations of multi-Pomeron exchanges at high energies. Naturally, all of these findings also hold in QCD, as was discussed in Refs. [70,80,83,106-108], and confirmed by experimental evidence (see also a more recent discussion in Ref. [29]).

The extension of the CGC/saturation framework to the description of high-multiplicity events is quite straightforward, as was discussed in Refs. [31,62,63,66-72,74]. In this paper we consider only multiplicity dependence in $p p$ collisions for energies not exceeding $\sqrt{s} \approx 13 \mathrm{TeV}$ available at the LHC, and for moderately large values of relative multiplicity enhancement $n \equiv N_{\mathrm{ch}} /\left\langle N_{\mathrm{ch}}\right\rangle \lesssim 10$, where $N_{\mathrm{ch}}$ and $\left\langle N_{\mathrm{ch}}\right\rangle$ are, respectively, the number of charged particles observed in a given multiplicity class and the average over all multiplicity classes in a given experimental setup. In this kinematics the density of produced particles is still too low for the formation of dense hot QGP, as it happens in heavyion collisions [7-21]. Due to the apparent similarity of heavy-ion and large-multiplicity events, in the literature it is sometimes expected that certain effects which were extensively studied in the context of heavy-ion collisions (e.g., energy loss effect; see Refs. [20,109-138]) might also be relevant in very high-multiplicity $p p$ collisions. As was found by two independent studies [139,140], for $p p$ collisions in the LHC kinematics these effects do not exceed ten percent for $n \lesssim 10$, within the uncertainty of theoretical predictions and experimental errors of available data. For this reason, in what follows we may disregard all such interactions and, in view of the local parton hadron duality hypothesis [141-143], assume that the multiplicity of produced hadrons in a given event is directly proportional to the number of partons produced in a collision.

The probability of multiplicity fluctuations decreases rapidly as a function of the number of produced charged particles $N_{\text {ch }}$ [144], and therefore to study the multiplicity dependence it is more common to use a self-normalized ratio [100], 


$$
\frac{d N_{M} / d y}{\left\langle d N_{M} / d y\right\rangle}=\frac{d \sigma_{M}(y, \eta, \sqrt{s}, n) / d y}{d \sigma_{M}(y, \eta, \sqrt{s},\langle n\rangle=1) / d y} / \frac{d \sigma_{\mathrm{ch}}\left(\eta, \sqrt{s}, Q^{2}, n\right) / d \eta}{d \sigma_{\mathrm{ch}}\left(\eta, \sqrt{s}, Q^{2},\langle n\rangle=1\right) / d \eta},
$$

where $d \sigma_{M}(y, \eta, \sqrt{s}, n)$ is the cross section of strange hadron $M$ production, having rapidity $y$ and accompanied by $N_{\mathrm{ch}}=n\left\langle N_{\mathrm{ch}}\right\rangle$ charged particles with rapidity $\eta$, and $d \sigma_{\mathrm{ch}}(\eta, \sqrt{s}, n)$ is the total production cross section for $N_{\mathrm{ch}}=n\left\langle N_{\mathrm{ch}}\right\rangle$ charged particles with the same rapidity $\eta$. Since the cross sections are proportional to the probability to produce a given final state, the ratio (17) might be interpreted as a conditional probability to produce a strange hadron $M$ in a $p p$ collision in which $N_{\text {ch }}$ charged particles are produced.

We expect that even in high-multiplicity events each dipole amplitude should satisfy the nonlinear BalitskyKovchegov equation, and thus might be approximately described by Eq. (12), although the value of the saturation scale $Q_{s}$ might be modified. As was demonstrated in Refs. [66-68], the observed charged multiplicity $d N_{\mathrm{ch}} / d y$ of soft hadrons in $p p$ collisions is proportional to the saturation scale $Q_{s}^{2}$ (modulo logarithmic corrections), and therefore in the dipole framework the events with large multiplicity might be described by simply rescaling $Q_{s}^{2}$ as a function of $n$ [66-72],

$$
Q_{s}^{2}(x, b ; n) \approx n Q^{2}(x, b) .
$$

The accuracy of the approximation (18) was tested in Ref. [63], and it was found that its error does not exceed 10 percent in the region of interest $(n \lesssim 10)$, on par with the precision of current evaluations. Therefore, in what follows we will use Eq. (18) for our estimates. While at LHC energies it is expected that the typical values of the saturation scale $Q_{s}(x, b)$ fall within the range $0.5-1 \mathrm{GeV}$, from Eq. (18) we can see that in events with enhanced multiplicity this parameter might significantly exceed this estimate, leading to an interplay of the large- $Q_{s}$ and large- $p_{T}$ limits. Since increasing multiplicity and increasing energy (decreasing $x$ ) affect $Q_{s}^{2}$ in a similar way, the study of the high-multiplicity events allows to study a deeply saturated regime, which determines the dynamics of all processes at significantly higher energies. In the large$p_{T}$ kinematics the typical sizes of the dipoles are small, $r \sim 1 / p_{T}$, so we may expect from Eqs. (12) and (18) that the dependence of the dipole amplitude on multiplicity simplifies and is given by the multiplicative factor $\sim n^{\left\langle\gamma_{\text {eff }}\right\rangle}$, where $n$ is the relative enhancement of multiplicity, and the value of the parameter $\gamma_{\mathrm{eff}}$ is given in Eq. (14).

In inclusive strange hadron production the accompanying charged particles might be produced with rapidities both below and above that of strange hadrons. In the dipole picture this implies that we should share the observed enhanced multiplicity between the dipole amplitude (8) and gluon PDF (9)-(11) which appear in Eq. (2). As explained in Fig. 4, this suggests that the observed multiplicity dependence should depend on the experimental setup, namely, if the detector can distinguish charged particles produced with rapidities either above or below that of a strange hadron. For the most widely used symmetric

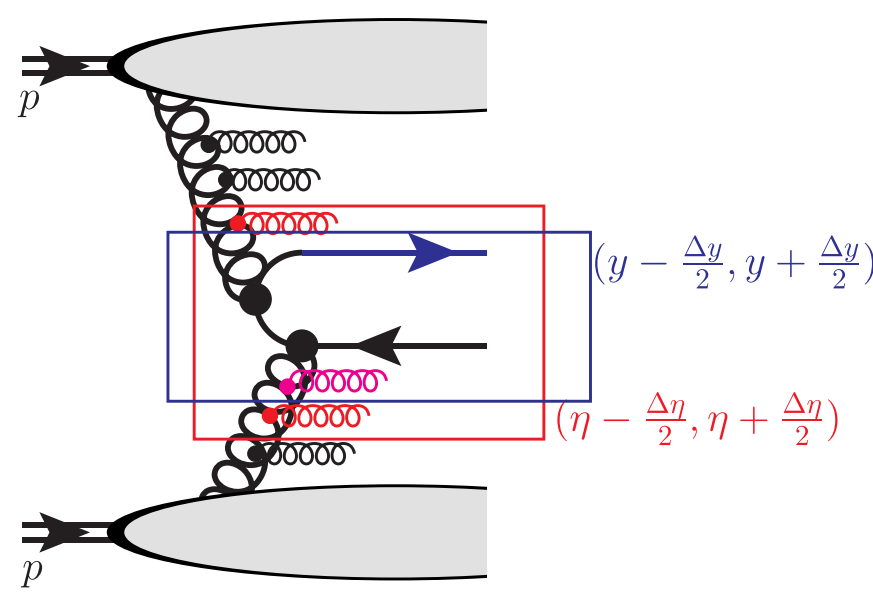

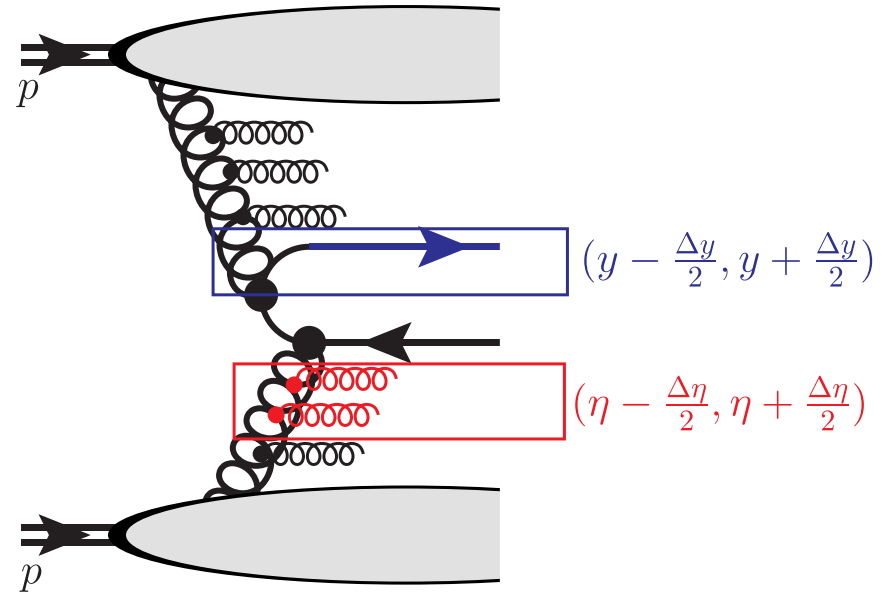

FIG. 4. Demonstration that measured multiplicity depends on the experimental setup. For the sake of plot legibility, we have replaced the dipole amplitudes with Balitsky-Fadin-Kuraev-Lipatov-style Reggeized gluons (cut Pomerons). Left: Experimental setup when the rapidity bin used for the collection of strange hadrons (blue box) partially overlaps with the bin used for the collection of charged particles (red box). In this kinematics the charged particles might be produced with rapidities either above or below that of the strange meson. Right: Experimental setup in which the rapidity bins used for the collection of strange hadrons and charged particle do not overlap. The elevated multiplicity in this case should be unambiguously attributed to a dipole amplitude or gluon uPDF. 


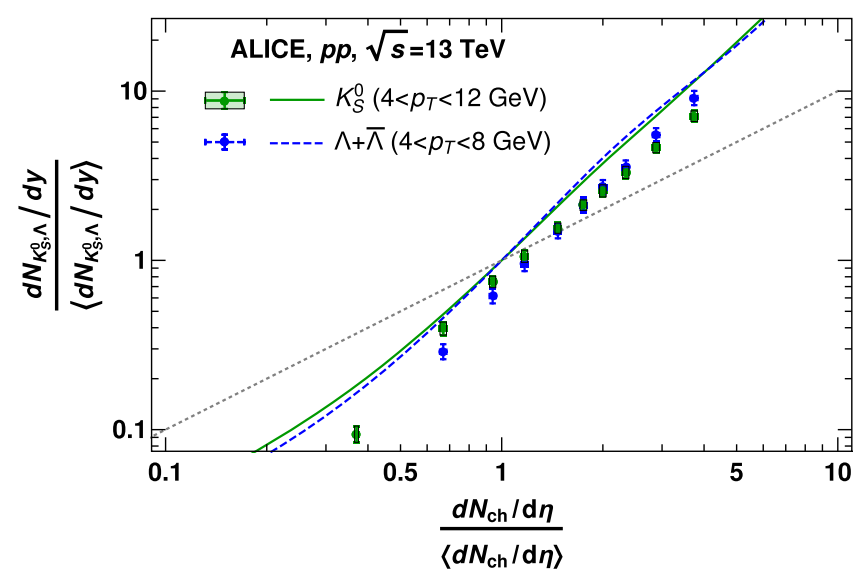

FIG. 5. Comparison of the theoretical multiplicity dependence for $K_{S}^{0}$ meson production (solid curve) and $\Lambda$ baryons (dashed curve) with experimental data from ALICE [100]. The almost linear dependence in double-log coordinates suggests that dependence on $n=\left(d N_{\mathrm{ch}} / d \eta\right) /\left\langle d N_{\mathrm{ch}} / d \eta\right\rangle$ is approximately power-like, $\sim n^{\alpha}$. The charged particles and strange hadrons are collected at central rapidities. For the sake of reference, we also show a dotted line, which corresponds to a linear dependence.

configuration $|\eta|,|y| \leq 1$, we should average over all possible partitions of the observed number of charged particles. This evaluation is technically quite complicated, although it finally leads to an intuitive result that predominantly the multiplicity enhancement is shared equally [73]. For this reason, we expect for production at central rapidities that the multiplicity dependence of the cross section should be $\sim(n / 2)^{2\left\langle\gamma_{\text {eff }}\right\rangle}$. However, the multiplicity dependence would be different when strange hadrons and charged particles are collected in well-separated rapidity bins. As can be seen from the right panel of Fig. 4, in that case the enhanced multiplicity should be assigned only to a dipole amplitude, so we expect that multiplicity dependence will be much milder, $\sim n^{\left\langle\gamma_{\text {eff }}\right\rangle}$.

In Fig. 5 we compare the theoretical expectations for the multiplicity dependence of strange hadrons $\left(K_{S}^{0}\right.$ and $\Lambda$ ) with experimental data from the ALICE Collaboration [100]. These data were collected at central rapidities in the bins with large transverse momenta $p_{T} \gtrsim 4 \mathrm{GeV}$, where the color dipole framework is well justified. We can see that our approach can describe the slope of the experimentally observed $n$ dependence reasonably well, and agrees with our expectations from the previous paragraph in the large- $p_{T}$ kinematics. However, our theoretical curves overestimate all of the experimental points by the same normalization factor 1.2 . By definition, the self-normalized ratio (17) should equal one at the point $n=1$. This condition is fulfilled for our theoretical curves but, surprisingly, not for the experimental data. For this reason, we believe that the normalization of our curves is correct.

\section{CONCLUSIONS}

In this paper we studied the production of strange hadrons in the color dipole approach. We found that the CGC/saturation approach can describe the cross sections in the large- $p_{T}$ kinematics, although it might not be very reliable for smaller $p_{T}$. The latter restriction implies that the suggested approach cannot be applied to $p_{T}$-integrated observables, which get their dominant contribution from the nonperturbative small- $p_{T}$ region.

We also applied the CGC/saturation approach to the description of the multiplicity dependence measured by ALICE [100]. These data were collected at sufficiently large transverse momenta $p_{T} \gtrsim 4 \mathrm{GeV}$ of kaons and $\Lambda$ baryons, where our approach is well justified. We found that the theoretical expectations for multiplicity dependence are in reasonable agreement with experimental data. Our evaluation is largely parameter free and relies only on the choice of the parametrization for the dipole cross section (12) and fragmentation functions of strange hadrons. This study complements our previous analyses of the multiplicity dependence of heavier charm and bottom production [62] and demonstrates that at sufficiently large $p_{T}$ it is possible to describe them all within the same framework.

Finally, we need to mention that the suggested approach is valid for energies not exceeding $\sqrt{s} \approx 13-14 \mathrm{TeV}$ available at the LHC, and for moderately large values of $n \equiv N_{\text {ch }} /\left\langle N_{\text {ch }}\right\rangle \lesssim 10$. For significantly larger energies, e.g., at the planned Future Circular Collider [145], we expect that the density of charged particles will increase drastically, leading to the formation of dense hot QGP [7-21], even in $p p$ collisions. The interactions with dense matter in that kinematics will give rise to a plethora of new effects and production mechanisms [20,109-131,133-138].

\section{ACKNOWLEDGMENTS}

We thank our colleagues at UTFSM University for encouraging discussions. This research was partially supported by Project No. ANID PIA/APOYO AFB180002 (Chile) and Fondecyt (Chile) Grant No. 1180232. Also, we thank Yuri Ivanov for technical support of the USM HPC cluster, where part of the evaluations were performed.

\section{APPENDIX A: FRAGMENTATION FUNCTIONS}

In this Appendix we would like to briefly summarize the fragmentation functions used in our evaluations. These functions are nonperturbative objects, which cannot be evaluated from first principles. For this reason, currently their parametrization is extracted from the phenomenological fits of experimental data. For the sake of definiteness, for our evaluations we use the fragmentation functions for kaons and $\Lambda$ from Ref. [99] (the so-called AKK08 parametrization). The fragmentation functions for $K^{ \pm}$and $K_{S}^{0}$ are constrained by the isospin symmetry relation 

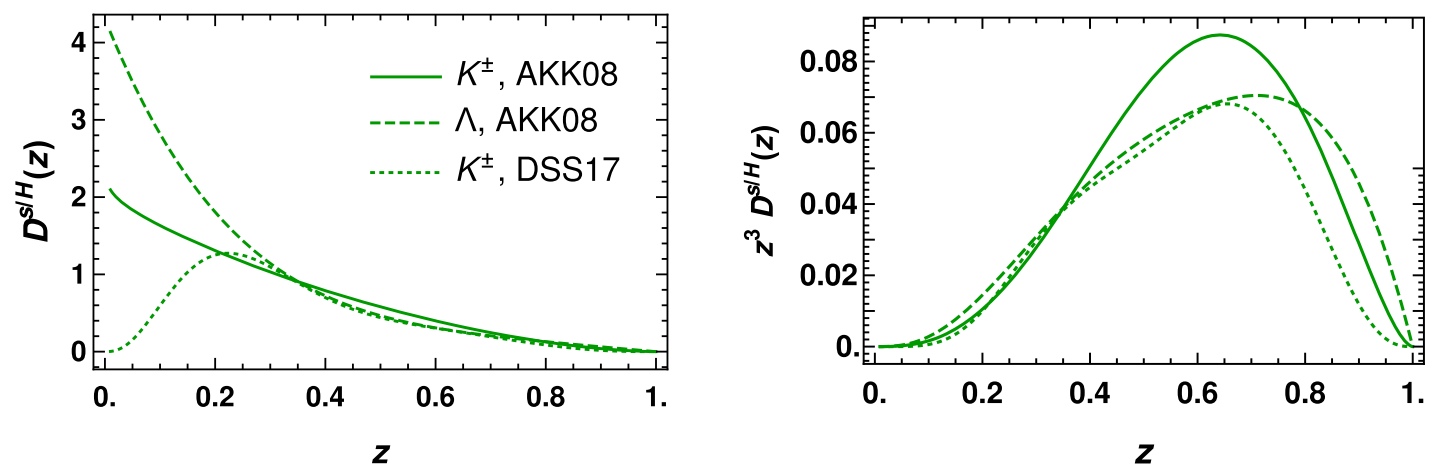

FIG. 6. Left: Fragmentation function $D^{s / H}$ of kaons and $\Lambda$ baryons ( $s$-quark component), evaluated in the AKK08 [99] and DSS17 [146] parametrizations. Right: The same function multiplied by $z^{3}$. As explained in the text, this factor $\sim z^{3}$ appears in the physical cross section in the large- $p_{T}$ kinematics and effectively suppresses the differences of fragmentation functions in the small- $z$ domain, which are seen in the left panel.

$$
D_{i}^{K_{S}^{0}}\left(z, \mu^{2}\right)=\frac{1}{2} D_{\bar{i}}^{K^{ \pm}}\left(z, \mu^{2}\right),
$$

and therefore in what follows we may consider only the fragmentation function of neutral kaons $K_{S}^{0}$. For kaons we checked that the alternative parametrizations of fragmentation functions-DSS17 [146], NNPDF [147], and JAM [148] — give similar results in the region of interest. We have not found parametrizations for fragmentation functions of strange baryons $\Omega, \Xi$ or for $K_{S}^{0 *}$ and $\phi$ mesons, and for this reason we do not consider them in this paper.

In the AKK08 parametrization [99] it is assumed that the fragmentation function is given by

$$
D^{i / H}(z)=N_{i} z^{a_{i}}(1-z)^{b_{i}}\left[1+c_{i}(1-z)^{d_{i}}\right],
$$

where $N_{i}, a_{i}, b_{i}, c_{i}, d_{i}$ are some numerical coefficients which depend on the hadron and quark flavor $i$. We expect that for strange hadrons the largest contribution comes from the fragmentation of the strange quark, and thus below we will discuss the fragmentation function $D^{s / H}$. As we can see from Fig. 6, the parametrizations for $K^{ \pm}$mesons and $\Lambda$ baryons differ quite substantially in the region of small $z \lesssim 0.3$, although they become comparable for all hadrons at larger values of $z$.

In this paper we are mostly interested in the large- $p_{T}$ kinematics, and it is possible to show that this region has stronger sensitivity to the region of large $z$. Indeed, as we can see from the structure of Eq. (2), at large $p_{T}$ the cross section $d \sigma_{\bar{Q} Q} / d p_{T}^{\bar{Q} Q}$ is suppressed as $\sim\left(1 / p_{T}^{\bar{Q} Q}\right)^{n}$ with $n \gtrsim 5$. The momentum of the quark pair $p_{T}^{\bar{Q} Q}$ is related to the momentum of the strange hadron as $p_{T}^{\bar{Q} Q}=p_{T} / z$, so this implies that in the integral over the fragmentation fraction $z$ in Eq. (1) effectively we get an additional prefactor $\sim z^{n-2}$, which suppresses the contribution of the small- $z$ domain. As we can see from the right panel of Fig. 6, the dominant contribution comes from the region $z \sim 0.6-0.8$, where the difference between fragmentation functions does not exceed a factor of 2 .

\section{APPENDIX B: EVALUATION OF THE DIPOLE AMPLITUDES}

In this Appendix, for the sake of completeness, we briefly remind the reader about the main technical steps and assumptions used in Refs. $[57,58,61]$ for the derivation of the cross section (8). This derivation follows the general rules for the evaluation of the hard amplitudes in terms of the color-singlet processes introduced in Refs. [50,52-58]. For the sake of definiteness we will follow the procedure and notations as discussed in Refs. [57,58,61] [105]. Although this derivation was suggested for heavy quarks, we expect that in the kinematics of large transverse momenta $p_{T}$, the latter variable will play the same role as the heavy quark mass in Refs. $[57,58,61]$, thus justifying the extension of those techniques to the strange sector. For example, we expect that the typical size of dipoles is small, $r \sim 1 / p_{T}$, so the interaction with perturbative gluons is suppressed at least as $\sim \alpha_{s}\left(p_{T}\right) / p_{T}^{2}$. However, in LHC kinematics, due to saturation effects we expect that the gluonic fields are enhanced, so each gluon should be understood as a parton shower (a so-called "BK Pomeron"). Since the interactions of quarks and antiquarks at high energies do not change the helicities of fermions, the effective interaction of the quark with the gluonic field of the proton might be described by a factor $\pm i g t^{a} \gamma_{a}\left(\boldsymbol{x}_{\perp}\right)$, where $\boldsymbol{x}_{\perp}$ is the transverse coordinate of the quark, and the nonperturbative function $\gamma\left(\boldsymbol{x}_{\perp}\right)$ is related to a distribution of gluons in the target. The assumption that the interaction of the dipole with the target is described by the same color group generator $t_{a}$ as in perturbative QCD (pQCD) constitutes the central assumption of the approach $[57,58,61]$, and is valid only for hard processes like, e.g., pQCD. The relation of $\gamma_{a}\left(\boldsymbol{x}_{\perp}\right)$ to the dipole scattering amplitude 
$N(x, \boldsymbol{r})$ may be found from photon-induced deep inelastic scattering, and is given by

$N(x, \boldsymbol{r})=\frac{1}{8} \int d^{2} b\left|\gamma_{a}(x, \boldsymbol{b}-z \boldsymbol{r})-\gamma_{a}(x, \boldsymbol{b}+\bar{z} \boldsymbol{r})\right|^{2}$,

where $\boldsymbol{r} \equiv \boldsymbol{x}_{Q}-\boldsymbol{x}_{\bar{Q}}$ is the transverse size of the dipole, and $z$ is the light-cone fraction of the dipole momentum carried by the quarks.

In the Iancu-Mueller approach [149] (see also Ref. [66]) it was shown that the interaction of the dipole with the target is described by the $S$-matrix element

$$
S\left(y, \boldsymbol{x}_{Q}, \boldsymbol{x}_{\bar{Q}}\right)=\frac{1}{N_{c}}\left\langle\operatorname{tr}\left(V^{\dagger}\left(\boldsymbol{x}_{\boldsymbol{Q}}\right) V\left(\boldsymbol{x}_{\bar{Q}}\right)\right)\right\rangle,
$$

where $y=\ln (1 / x)$ is the rapidity of the dipole, and $V^{\dagger}\left(\boldsymbol{x}_{Q}\right)$ and $V\left(\boldsymbol{x}_{\bar{Q}}\right)$ in Eq. (B2) are Wilson lines describing the scattering of a quark and antiquark with transverse coordinates $\boldsymbol{x}_{Q}, \boldsymbol{x}_{\bar{Q}}$ in the color field of a hadron,

$$
V\left(\boldsymbol{x}_{\perp}\right)=P \exp \left(i g \int d x^{-} A_{a}^{+}\left(x^{-}, \boldsymbol{x}_{\perp}\right) t^{a}\right),
$$

where $A_{\mu}^{a}$ is the gluonic field in a hadron. The dipole amplitude $N(x, \boldsymbol{r})$ is related to $S\left(y, \boldsymbol{x}_{Q}, \boldsymbol{x}_{\bar{Q}}\right)$ as

$$
N(x, \boldsymbol{r})=1-S\left(y, \boldsymbol{x}_{Q}, \boldsymbol{x}_{\bar{Q}}\right) .
$$

As we discussed earlier, for very small dipoles of size $r \sim$ $1 / p_{T}$ we may expect that the interaction of a dipole with the gluonic field becomes perturbative, so we can see that Eqs. (B2)-(B4) might be approximated in this limit by the first $\mathcal{O}\left(g^{2}\right)$ term, which coincides with Eq. (B1), provided $\gamma_{a}(\boldsymbol{x})$ is identified with a gluonic field $\sim g \int d x^{-} A_{a}^{+}\left(x^{-}, \boldsymbol{x}\right)$. At the same time, we do not assume that the interaction of the gluons with each other, as well as with light quarks in general, is perturbative, so we expect that the dipole amplitude should still satisfy the nonlinear BalitskyKovchegov equation.

Equation (B1) might be rewritten in the form

$$
\frac{1}{8} \int d^{2} \boldsymbol{b} \gamma_{a}(x, \boldsymbol{b}) \gamma_{a}(x, \boldsymbol{b}+\boldsymbol{r})=\frac{1}{2} N(x, \boldsymbol{r})+\underbrace{\int d^{2} b\left|\gamma_{a}(x, \boldsymbol{b})\right|^{2}}_{=\text {const }} .
$$

The value of the constant term on the right-hand side of Eq. (B5) is related to the infrared behavior of the theory, and for the observables that we consider in this paper it cancels exactly. Using Eq. (B5), we can rewrite the production cross sections of certain processes in terms of the dipole amplitudes $N(x, \boldsymbol{r})$ with different arguments.

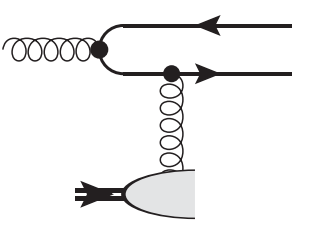

(a)

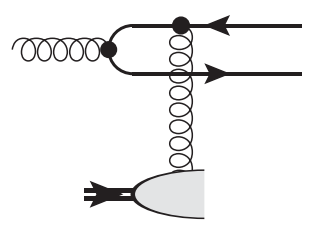

(b)

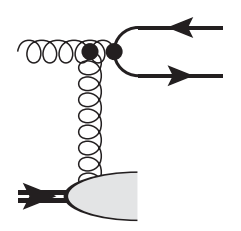

(c)
FIG. 7. Diagrams that contribute to the meson production cross section in the leading order of perturbative QCD. The contribution of the diagram $(c)$ to the meson formation might also be viewed as gluon-gluon fusion $g g \rightarrow g$, with subsequent gluon fragmentation $g \rightarrow \bar{Q} Q \rightarrow \mathcal{M}+X$. In the color dipole approach gluons are replaced with a combination of color-singlet dipole amplitudes, as explained in the text.

For the leading-order hadroproduction of the $\bar{Q} Q$ pair we should take the diagrams shown in Fig. 7. As was demonstrated in Refs. [57,58,61], this evaluation yields for the amplitude of the process $g p \rightarrow \bar{Q} Q X$

$$
\begin{aligned}
& \mathcal{A}_{a}(z, \boldsymbol{b}, \boldsymbol{r}) \\
& =\left[\left(\frac{\delta_{a b}}{6}+\frac{d_{a b c}}{2} t_{c}\right)\left[\gamma_{b}(x, \boldsymbol{b}-z \boldsymbol{r})-\gamma(x, \boldsymbol{b}+\bar{z} \boldsymbol{r})\right]\right. \\
& \left.\quad+\frac{i f_{a b c}}{2} t_{c}\left[\gamma_{b}(x, \boldsymbol{b}-z \boldsymbol{r})+\gamma_{b}(x, \boldsymbol{b}+\bar{z} \boldsymbol{r})-2 \gamma_{b}(x, \boldsymbol{b})\right]\right] \\
& \quad \times \Psi_{\bar{Q} Q}(\boldsymbol{r}, z),
\end{aligned}
$$

where $\Psi_{\bar{Q} Q}$ is the $\bar{Q} Q$ component of the gluon wave function, and $t_{a}$ are color generators in the fundamental representation. As we can see, in the limit $r \rightarrow 0$ the amplitude vanishes, as expected from color transparency. While for the color singlet $\bar{Q} Q$ such cancellation is obvious, for the color-octet part this result is less trivial and happens due to contribution of the diagram (c) in Fig. 7, as given by the last term $\sim \gamma_{b}(x, \boldsymbol{b})$ in the second line of Eq. (B6). We can see that both color-singlet and color-octet amplitudes vanish in the limit of small dipoles $(r \rightarrow 0)$.

For the $p_{T}$-dependent cross section we should project the produced quark onto the states with definite transverse momentum $\boldsymbol{p}_{T}$, both in the amplitude and its conjugate, and integrate over the kinematics of the produced antiquark $\bar{Q}$ (which leads to the equality of transverse coordinates of $\bar{Q}$ in the amplitude and conjugate). After straightforward simplifications, we get for the square of the amplitude

$$
\begin{aligned}
\left|\mathcal{A}_{g p \rightarrow Q X}\right|^{2} \sim & \int d z d^{2} \boldsymbol{r}_{1} d^{2} \boldsymbol{r}_{2} \int d^{2} \boldsymbol{b}_{1} e^{-i \boldsymbol{p}_{T}\left(\boldsymbol{r}_{1}-\boldsymbol{r}_{2}\right)} \\
& \times\left\langle\mathcal{A}^{\dagger}\left(z, \boldsymbol{b}_{2}, \boldsymbol{r}_{2}\right) \mathcal{A}_{a}\left(z, \boldsymbol{b}_{1}, \boldsymbol{r}_{1}\right)\right\rangle_{\boldsymbol{b}_{2}=\boldsymbol{b}_{1}+\bar{z} \boldsymbol{r}_{1}-\bar{z} \boldsymbol{r}_{2}}
\end{aligned}
$$

where the subscript index $i$ takes values $i=1,2$ and distinguishes variables in the amplitude and its conjugate. 
For the evaluation of the integral over $\boldsymbol{b}_{1}$ we may use Eq. (B5), and after some algebraic simplifications obtain Eqs. (2)-(8). While in this Appendix we assumed that the transverse momentum of the primordial gluon is zero, we may take into account possible nonzero transverse components via additional convolution with the momentum distribution of the incident ("primordial") gluons, as appears in the first line of Eq. (2).
[1] T. Alber et al., Z. Phys. C 64, 195 (1994).

[2] E. Anderson et al., Phys. Lett. B 294, 127 (1992); 327, 433 (1994).

[3] S. Abatzis et al., Nucl. Phys. A566, 499c (1994); Phys. Lett. B 316, 615 (1993).

[4] D. DiBari et al., Nucl. Phys. A590, 307c (1995).

[5] J. B. Kinson et al., Nucl. Phys. A590, 317 (1995).

[6] M. Gazdzicki et al., Nucl. Phys. A590, 197c (1995).

[7] J. Rafelski and B. Muller, Phys. Rev. Lett. 48, 1066 (1982); 56, 2334(E) (1986).

[8] J. Rafelski, Phys. Rep. 88, 331 (1982), https://inspirehep .net/literature/177741.

[9] P. Koch, B. Muller, and J. Rafelski, Phys. Rep. 142, 167 (1986).

[10] J. Letessier, A. Tounsi, U. W. Heinz, J. Sollfrank, and J. Rafelski, Phys. Rev. Lett. 70, 3530 (1993).

[11] J. Letessier, J. Rafelski, and A. Tounsi, Phys. Lett. B 328, 499 (1994).

[12] J. Rafelski and M. Danos, Phys. Rev. C 50, 1684 (1994).

[13] J. Sollfrank, M. Gazdzicki, U. Heinz, and J. Rafelski, Z. Phys. C 61, 659 (1994).

[14] J. Letessier, A. Tounsi, U. Heinz, J. Sollfrank, and J. Rafelski, Phys. Rev. D 51, 3408 (1995).

[15] P. Braun-Munzinger, J. Stachel, J. P. Wessels, and N. Xu, Phys. Lett. B 344, 43 (1995).

[16] J. W. Harris and B. Muller, Annu. Rev. Nucl. Part. Sci. 46, 71 (1996).

[17] P. Koch, B. Muller, and J. Rafelski, Int. J. Mod. Phys. A 32, 1730024 (2017).

[18] J. Rafelski and B. Muller, Phys. Rev. Lett. 48, 1066 (1982); 56, 2334(E) (1986).

[19] J. Rafelski, Eur. Phys. J. Special Topics 229, 1 (2020).

[20] B. Z. Kopeliovich, I. Schmidt, and M. Siddikov, Phys. Rev. C 95, 065203 (2017).

[21] I. Schmidt, M. Siddikov, and M. Musakhanov, Phys. Rev. C 98, 025207 (2018).

[22] B. Abelev et al. (ALICE Collaboration), Phys. Lett. B 728, 25 (2014).

[23] J. Adam et al. (ALICE Collaboration), Phys. Lett. B 758, 389 (2016).

[24] J. Adam et al. (ALICE Collaboration), Nat. Phys. 13, 535 (2017).

[25] N. Fischer and T. Sjöstrand, J. High Energy Phys. 01 (2017) 140.

[26] H. J. Pirner, B. Z. Kopeliovich, and K. Reygers, Phys. Rev. D 101, 114010 (2020).

[27] D. Thakur (ALICE Collaboration), Springer Proc. Phys. 234, 217 (2019).
[28] B. Abelev et al. (ALICE Collaboration), Phys. Lett. B 712, 165 (2012).

[29] E. Gotsman and E. Levin, Eur. Phys. J. C 81, 99 (2021).

[30] J. Adam et al. (ALICE Collaboration), J. High Energy Phys. 09 (2015) 148.

[31] M. Siddikov and I. Schmidt, Phys. Rev. D 102, 076020 (2020).

[32] M. Siddikov and I. Schmidt, Phys. Rev. D 104, 016023 (2021).

[33] G. T. Bodwin, E. Braaten, and G. P. Lepage, Phys. Rev. D 51, 1125 (1995); 55, 5853(E) (1997).

[34] F. Maltoni, M. L. Mangano, and A. Petrelli, Nucl. Phys. B519, 361 (1998).

[35] N. Brambilla, A. Vairo, and E. Mereghetti, Phys. Rev. D 79, 074002 (2009); 83, 079904(E) (2011).

[36] Y. Feng, J. P. Lansberg, and J. X. Wang, Eur. Phys. J. C 75, 313 (2015).

[37] N. Brambilla et al., Eur. Phys. J. C 71, 1534 (2011).

[38] S. P. Baranov, A. V. Lipatov, and N. P. Zotov, Eur. Phys. J. C 75, 455 (2015).

[39] S. P. Baranov and A. V. Lipatov, Phys. Rev. D 96, 034019 (2017).

[40] R. Baier and R. Ruckl, Phys. Lett. 102B, 364 (1981).

[41] E. L. Berger and D. L. Jones, Phys. Rev. D 23, 1521 (1981).

[42] C. H. Chang, Nucl. Phys. B172, 425 (1980).

[43] R. Maciula and A. Szczurek, Phys. Rev. D 87, 094022 (2013).

[44] V. A. Abramovsky, V. N. Gribov, and O. V. Kancheli, Yad. Fiz. 18, 595 (1973), https://inspirehep.net/literature/ 84486.

[45] A. Capella and A. Kaidalov, Nucl. Phys. B111, 477 (1976).

[46] L. Bertocchi and D. Treleani, J. Phys. G 3, 147 (1977).

[47] Y. M. Shabelski, Sov. J. Nucl. Phys. 26, 573 (1977), https://inspirehep.net/literature/126444.

[48] N. N. Nikolaev and W. Schafer, Phys. Rev. D 74, 074021 (2006).

[49] A. B. Kaidalov and K. A. Ter-Martirosian, Phys. Lett. B 117, 247 (1982).

[50] L. V. Gribov, E. M. Levin, and M. G. Ryskin, Phys. Rep. 100, 1 (1983).

[51] L. D. McLerran and R. Venugopalan, Phys. Rev. D 49, 2233 (1994).

[52] L. D. McLerran and R. Venugopalan, Phys. Rev. D 49, 3352 (1994).

[53] L. D. McLerran and R. Venugopalan, Phys. Rev. D 50, 2225 (1994).

[54] A. H. Mueller and J. Qiu, Nucl. Phys. B268, 427 (1986). 
[55] L. McLerran and R. Venugopalan, Phys. Rev. D 49, 3352 (1994); Phys. Rev. D 50, 2225 (1994); Phys. Rev. D 59, 094002 (1999).

[56] K. J. Golec-Biernat and M. Wusthoff, Phys. Rev. D 60, 114023 (1999).

[57] B. Z. Kopeliovich and A. V. Tarasov, Nucl. Phys. A710, 180 (2002).

[58] B. Kopeliovich, A. Tarasov, and J. Hufner, Nucl. Phys. A696, 669 (2001).

[59] J. Binnewies, B. A. Kniehl, and G. Kramer, Phys. Rev. D 58, 034016 (1998).

[60] B. A. Kniehl and G. Kramer, Phys. Rev. D 60, 014006 (1999).

[61] V. P. Goncalves, B. Kopeliovich, J. Nemchik, R. Pasechnik, and I. Potashnikova, Phys. Rev. D 96, 014010 (2017).

[62] I. Schmidt and M. Siddikov, Phys. Rev. D 101, 094020 (2020).

[63] Y. Q. Ma, P. Tribedy, R. Venugopalan, and K. Watanabe, Phys. Rev. D 98, 074025 (2018).

[64] Y. V. Kovchegov, Phys. Rev. D 61, 074018 (2000).

[65] V. Barone, M. Genovese, N. N. Nikolaev, E. Predazzi, and B. G. Zakharov, Phys. Lett. B 326, 161 (1994).

[66] Y. V. Kovchegov and E. Levin, Quantum Chromodynamics at High Energy, Vol. 33 (Cambridge University Press, Cambridge, England, 2012).

[67] D. Kharzeev and M. Nardi, Phys. Lett. B 507, 121 (2001); D. Kharzeev and E. Levin, Phys. Lett. B 523, 79 (2001), D. Kharzeev, E. Levin, and M. Nardi, Phys. Rev. C 71, 054903 (2005), J. Phys. G 35, 054001 (2008).

[68] A. Dumitru, D. E. Kharzeev, E. M. Levin, and Y. Nara, Phys. Rev. C 85, 044920 (2012).

[69] D. Kharzeev and M. Nardi, Phys. Lett. B 507, 121 (2001).

[70] Y. V. Kovchegov, Nucl. Phys. A692, 557 (2001).

[71] E. Levin and A. H. Rezaeian, Phys. Rev. D 82, 014022 (2010).

[72] T. Lappi, Eur. Phys. J. C 71, 1699 (2011).

[73] E. Levin and M. Siddikov, Eur. Phys. J. C 79, 376 (2019).

[74] M. Siddikov, E. Levin, and I. Schmidt, Eur. Phys. J. C 80, 560 (2020).

[75] V. A. Khoze, A. D. Martin, M. G. Ryskin, and W. J. Stirling, Eur. Phys. J. C 39, 163 (2005).

[76] L. Motyka and M. Sadzikowski, Eur. Phys. J. C 75, 213 (2015).

[77] I. Schmidt and M. Siddikov, J. Phys. G 46, 065002 (2019).

[78] E. Levin, L. Lipatov, and M. Siddikov, Phys. Rev. D 94, 096004 (2016).

[79] N. N. Nikolaev and B. G. Zakharov, J. Exp. Theor. Phys. 78, 598 (1994), https://inspirehep.net/literature/383552.

[80] Y. V. Kovchegov, Phys. Rev. D 60, 034008 (1999).

[81] Y. V. Kovchegov and H. Weigert, Nucl. Phys. A784, 188 (2007).

[82] I. Balitsky and G. A. Chirilli, Phys. Rev. D 77, 014019 (2008).

[83] Y. V. Kovchegov and E. Levin, Cambridge Monogr. Part. Phys., Nucl. Phys., Cosmol. 33, 1 (2012).

[84] I. Balitsky, Phys. Lett. B 518, 235 (2001).

[85] F. Cougoulic and Y. V. Kovchegov, Phys. Rev. D 100, 114020 (2019).
[86] C. A. Aidala et al., Probing Nucleons and Nuclei in High Energy Collisions (World Scientific, Singapore, 2020), https://doi.org/10.1142/11684.

[87] Y. Q. Ma and R. Venugopalan, Phys. Rev. Lett. 113, 192301 (2014).

[88] We would like to remind the reader that in the case of $D$ - and $B$-meson production studied earlier in Refs. [59-63], the scale in the small- $p_{T}$ kinematics was set by the heavy quark mass, so the use of the framework was justified up to $p_{T} \approx 0$. In case of the strangeness production this is no longer true, and we have to restrict our consideration to the large- $p_{T}$ domain only.

[89] H. G. Dosch, T. Gousset, G. Kulzinger, and H. J. Pirner, Phys. Rev. D 55, 2602 (1997).

[90] J. D. Bjorken, J. B. Kogut, and D. E. Soper, Phys. Rev. D 3, 1382 (1971).

[91] Y. V. Kovchegov and K. Tuchin, Phys. Rev. D 74, 054014 (2006).

[92] M. A. Kimber, A. D. Martin, and M. G. Ryskin, Phys. Rev. D 63, 114027 (2001).

[93] R. S. Thorne, AIP Conf. Proc. 792, 324 (2005).

[94] E. Iancu, K. Itakura, and S. Munier, Phys. Lett. B 590, 199 (2004).

[95] H. Kowalski and D. Teaney, Phys. Rev. D 68, 114005 (2003).

[96] H. Kowalski, L. Motyka, and G. Watt, Phys. Rev. D 74, 074016 (2006).

[97] G. Watt and H. Kowalski, Phys. Rev. D 78, 014016 (2008).

[98] A. H. Rezaeian and I. Schmidt, Phys. Rev. D 88, 074016 (2013).

[99] S. Albino, B. A. Kniehl, and G. Kramer, Nucl. Phys. B803, 42 (2008).

[100] S. Acharya et al. (ALICE Collaboration), Eur. Phys. J. C 80, 167 (2020).

[101] V. Khachatryan et al. (CMS Collaboration), J. High Energy Phys. 05 (2011) 064.

[102] D. Acosta et al. (CDF Collaboration), Phys. Rev. D 72, 052001 (2005).

[103] B. I. Abelev et al. (STAR Collaboration), Phys. Rev. C 75, 064901 (2007).

[104] We also would like to mention that the CMS and STAR data were not normalized to unity. For example, the CMS data instead of $N_{\mathrm{ev}}$ used the number of non-singlediffractive events $N_{\mathrm{NSD}}$, so we also corrected the normalization of experimental data.

[105] Other approaches might differ in minor technical details, though finally give equivalent results.

[106] J. Bartels and M. G. Ryskin, Z. Phys. C 76, 241 (1997).

[107] J. Bartels, M. Salvadore, and G. P. Vacca, Eur. Phys. J. C 42, 53 (2005).

[108] T. Sjostrand and P.Z. Skands, J. High Energy Phys. 03 (2004) 053.

[109] Y. Xu et al. (STAR Collaboration), J. Phys. G 37, 094059 (2010).

[110] X. N. Wang, Phys. Rev. C 58, 2321 (1998).

[111] Y. L. Dokshitzer and D. E. Kharzeev, Phys. Lett. B 519, 199 (2001).

[112] S. Albino, B. A. Kniehl, and G. Kramer, Nucl. Phys. B725, 181 (2005). 
[113] J. Adams et al. (STAR Collaboration), Phys. Lett. B 616, 8 (2005).

[114] J. Adams et al. (STAR Collaboration), Phys. Lett. B 637, 161 (2006).

[115] B. I. Abelev et al. (STAR Collaboration), Phys. Rev. Lett. 97, 152301 (2006).

[116] B. I. Abelev et al. (STAR Collaboration), Phys. Lett. B 655, 104 (2007).

[117] W. Liu, C. M. Ko, and B. W. Zhang, Phys. Rev. C 75, 051901(R) (2007).

[118] W. Liu and R. J. Fries, Phys. Rev. C 77, 054902(R) (2008).

[119] S. Sapeta and U. A. Wiedemann, Eur. Phys. J. C 55, 293 (2008).

[120] J. Adams et al. (STAR Collaboration), Phys. Rev. Lett. 94, 062301 (2005).

[121] B. I. Abelev et al. (STAR Collaboration), Phys. Rev. Lett. 98, 192301 (2007).

[122] S. Wicks, W. Horowitz, M. Djordjevic, and M. Gyulassy, Nucl. Phys. A784, 426 (2007).

[123] N. Armesto, M. Cacciari, A. Dainese, C. A. Salgado, and U. A. Wiedemann, Phys. Lett. B 637, 362 (2006).

[124] D. de Florian, R. Sassot, and M. Stratmann, Phys. Rev. D 76, 074033 (2007).

[125] Q. Wang and X. N. Wang, Phys. Rev. C 71, 014903 (2005).

[126] S. S. Adler et al. (PHENIX Collaboration), Phys. Rev. Lett. 96, 032301 (2006).

[127] B. I. Abelev et al. (STAR Collaboration), Phys. Rev. Lett. 98, 192301 (2007); 106, 159902(E) (2011).

[128] M. Djordjevic, M. Gyulassy, R. Vogt, and S. Wicks, Phys. Lett. B 632, 81 (2006).

[129] S. Wicks, W. Horowitz, M. Djordjevic, and M. Gyulassy, Nucl. Phys. A784, 426 (2007).

[130] A. Dainese, C. Loizides, and G. Paic, Eur. Phys. J. C 38, 461 (2005).

[131] K. J. Eskola, H. Honkanen, H. Niemi, P. V. Ruuskanen, and S. S. Rasanen, Nucl. Phys. A747, 511 (2005).

[132] B. Z. Kopeliovich, J. Nemchik, I. K. Potashnikova, and I. Schmidt, Phys. Rev. C 86, 054904 (2012).

[133] R. Baier, D. Schiff, and B. G. Zakharov, Annu. Rev. Nucl. Part. Sci. 50, 37 (2000).
[134] M. Gyulassy, I. Vitev, X. N. Wang, and B. W. Zhang, in Quark-Gluon Plasma 3 (World Scientific, Singapore, 2004), pp. 123-191, https://doi.org/10.1142/9789812795533_ 0003.

[135] J. D. Bjorken, FERMILAB-PUB-82-59-THY, Batavia (1982), https://inspirehep.net/literature?sort=mostrecent $\&$ size $=25 \&$ page $=1 \& q=F E R M I L A B-P U B-82-059-T$.

[136] B. Z. Kopeliovich and F. Niedermayer, Nuclear screening in $J / \psi$ and Drell-Yan pair production, Technical Report No. JINR-E2-84-834, Dubna, 1984, http://inspirehep.net/ record/209857.

[137] B. Z. Kopeliovich, J. Nemchik, I. K. Potashnikova, M. B. Johnson, and I. Schmidt, Phys. Rev. C 72, 054606 (2005).

[138] B. Z. Kopeliovich, H. J. Pirner, I. K. Potashnikova, and I. Schmidt, Phys. Lett. B 697, 333 (2011).

[139] S. Vogel, P. B. Gossiaux, K. Werner, and J. Aichelin, Phys. Rev. Lett. 107, 032302 (2011).

[140] A. Nath and Mishra, arXiv:1905.06918.

[141] Y. L. Dokshitzer, V. A. Khoze, and S. I. Troyan, J. Phys. G 17, 1585 (1991).

[142] V. A. Khoze, W. Ochs, and J. Wosiek, in At The Frontier of Particle Physics (World Scientific, Singapore, 2001), pp. 1101-1194, https://doi.org/10.1142/9789812810458_ 0028.

[143] V. A. Khoze and W. Ochs, Int. J. Mod. Phys. A 12, 2949 (1997).

[144] B. Abelev et al. (ALICE Collaboration), Phys. Lett. B 712, 165 (2012).

[145] M. Mangano, Physics at the FCC-hh, a $100 \mathrm{TeV}$ pp collider, CERN Yellow Reports: Monographs, 3/2017; doi: 10.23731/CYRM-2017-003 [arXiv:1710.06353 [hepph]], ISBN: 9789290834533 (Print), 9789290834540 (eBook).

[146] D. de Florian, M. Epele, R. J. Hernandez-Pinto, R. Sassot, and M. Stratmann, Phys. Rev. D 95, 094019 (2017).

[147] V. Bertone, S. Carrazza, N. P. Hartland, E. R. Nocera, and J. Rojo (NNPDF Collaboration), Eur. Phys. J. C 77, 516 (2017).

[148] N. Sato, J. J. Ethier, W. Melnitchouk, M. Hirai, S. Kumano, and A. Accardi, Phys. Rev. D 94, 114004 (2016).

[149] E. Iancu and A. H. Mueller, Nucl. Phys. 730, 460 (2004). 